\title{
Review: inadequate data exist on prevention and treatment strategies for foot ulcer in diabetes mellitus
}

Mason J, O'Keeffe C, McIntosh A, et al. A systematic review of foot ulcer in patients with type 2 diabetes mellitus. I:

Prevention. Diabet Med 1999 Oct;16:801-12.

Mason J, O'Keeffe C, Hutchinson A, et al. A systematic review of foot ulcer in patients with type 2 diabetes mellitus. II:

Treatment. Diabet Med 1999 Nov;16:889-909.

QUESTION: What strategies are effective in the prevention and treatment of foot ulcer

in patients with diabetes mellitus?

\section{Data sources}

Studies from 1983 to 1998 were identified from the Cochrane Trials Register, Medline, EMBASE/Excerpta Medica, CINAHL, HealthStar, PsycLIT, Science Citation Index, Social Science Citation Index, Index to Scientific and Technical Conference Proceedings, the HMIC database, and SIGLE.

\section{Study selection}

Studies were selected if they concerned screening, management, prevention, or education relating to the foot care of people with type 1 or type 2 diabetes.

\section{Data extraction}

Data were extracted on study design, interventions, and outcomes.

\section{Main results}

In relation to the prevention of diabetic foot ulcer, the search identified 5 randomised controlled trials (RCTs) of shared care by specialists and general practitioners, care by a hospital diabetes clinic, and care by general practitioners for patients without foot complications; 6 RCTs of foot care education (foot hygiene, treatment of callus, awareness of fungal infections, and care of cutaneous injuries) for patients without foot complications, of which 1 of the larger studies showed that foot ulcers were prevented for patients who agreed to behavioural contracts and periodic reminders; 1 RCT of screening and intervention for patients with feet at increased risk for ulceration, which showed that screening and intervention identified ulcers sooner, treated them more effectively, and reduced amputations; and 2 RCTs of therapeutic footwear in patients at increased risk for ulceration, of which 1 study showed that therapeutic shoes reduced ulcer relapses and the rate of new ulcers.

In relation to treatment of diabetic foot ulcer, the search identified 4 RCTs of antibiotic treatment; 8 RCTs of dressings and topical agents; 2 RCTs of cultured human dermis; 1 RCT of total contact casting; 2 RCTs of hyperbaric oxygen therapy; 2 RCTs of ketanserin, of which the larger study showed a reduction in ulcer area with ketanserin treatment; 6 RCTs of growth factors, of which 3 of the larger studies showed better ulcer healing with growth factor treatment; 1 RCT of granulocyte colony stimulating factor; and 1 RCT of patient education. Most studies had inadequate sample size and follow up and different study designs and outcomes. Therefore, meta-analytic comparisons were inappropriate for making firm conclusions. Individual studies show benefits for high risk patients from well organised, multidisciplinary foot care with rapid referral to specialist teams for foot problems or their precursors.

\section{Conclusions}

Studies on the effectiveness of prevention and treatment strategies for foot ulcer in patients with diabetes mellitus lack consistency in design and outcomes. Although they support the effectiveness of multidisciplinary approaches, they provide inadequate evidence for making firm conclusions.
Sources of funding:

NHS Executive and the British Diabetic

Association.

For correspondence: Dr J Mason, Medicines Evaluation Group, Centre for Health Economics, University of York, Heslington, York YO1 5DD, UK Fax $+44(0) 1904$ 433644 .

\section{COMMENTARY}

Lower extremity complications, including foot ulcers and amputations, are unacceptably common among patients with diabetes mellitus. A range of educational and therapeutic interventions are currently recommended to address this problem. The comprehensive and methodologically sound systematic reviews by Mason $e t$ al are a valuable contribution for evidence-based decision making because they highlight our current understanding of the effectiveness of many of these approaches. Perhaps the most important finding of this review is the relative lack of evidence. Despite the enormous effect on quality of life and health costs associated with lower extremity complications, the authors were able to locate only 41 trials, most of which were relatively small or used surrogate outcomes.

Several messages come from this review. For prevention, identifying patients at high risk for foot ulcers and arranging for them to receive a multidisciplinary intervention (chiropody and hygiene maintenance, hosiery and protective shoes, and education on daily hygiene) appears to be both effective and cost effective. For patients with established foot ulcers, an increasing number of treatment options are becoming available, including total contact casting, topical growth factors, and systemic hyperbaric oxygen therapy. Published studies have shown clinically significant benefits from these interventions, but unfortunately few of the studies have been adequately replicated.

An important reduction in the rate of lower extremity amputations may be realised with improved implementation of currently available evidence, but that remains to be seen. What we really need are large, simple trials evaluating interventions that can be applied in a range of healthcare settings.

Dereck L Hunt, MD McMaster University Hamilton, Ontario, Canada 